\title{
Intra-articular collagenase injection increases range of motion in a rat knee flexion contracture model
}

This article was published in the following Dove Press journal:

Drug Design, Development and Therapy

\section{Kayleigh Wong' \\ Guy Trudel ${ }^{2}$ \\ Odette Laneuville ${ }^{3}$}

'Bone and Joint Research Laboratory, The Ottawa Hospital Rehabilitation Centre, Ottawa, Ontario, ${ }^{2}$ Department of Medicine, Bone and Joint Research Laboratory, The Ottawa Hospital Rehabilitation Centre, Ottawa, Ontario, ${ }^{3}$ Department of Biology, Faculty of Science, University of Ottawa, Ottawa, Ontario, Canada
Correspondence: Odette Laneuville Department of Biology, Faculty of Science, University of Ottawa, Gendron Hall Room 4-449, 30 Marie Curie, Ottawa ON KIN 6N5, Canada Tel + I 6I35625800 ext 4606 Email olaneuvi@uottawa.ca
Objectives: A knee joint contracture, a loss in passive range of motion (ROM), can be caused by prolonged immobility. In a rat knee immobilization flexion contracture model, the posterior capsule was shown to contribute to an irreversible limitation in ROM, and collagen pathways were identified as differentially expressed over the development of a contracture. Collagenases purified from Clostridium histolyticum are currently prescribed to treat Dupuytren's and Peyronie's contractures due to their ability to degrade collagen. The potential application of collagenases to target collagen in the posterior capsule was tested in this model.

Materials and methods: Rats had one hind leg immobilized, developing a knee flexion contracture. After 4 weeks, the immobilization device was removed, and the rats received one $50 \mu \mathrm{L}$ intra-articular injection of $0.6 \mathrm{mg} / \mathrm{mL}$ purified collagenase. Control rats were injected with only the buffer. After 2 weeks of spontaneous remobilization following the injections, ROM was measured with a rat knee arthrometer, and histological sections were immunostained with antibodies against rat collagen types I and III.

Results/conclusion: Compared with buffer-injected control knees, collagenase-treated knees showed increased ROM in extension by $8.0^{\circ} \pm 3.8^{\circ}$ ( $p$-value $\left.<0.05\right)$. Immunohistochemical analysis revealed an increase in collagen type III staining $(p<0.01)$ in the posterior capsule of collagenase-treated knees indicating an effect on the extracellular matrix due to the collagenase. Collagen I staining was unchanged $(p>0.05)$. The current study provides experimental evidence for the pharmacological treatment of knee flexion contractures with intra-articular collagenase injection, improving the knee ROM.

Keywords: joint, contracture, collagen, immobilization range of motion

\section{Introduction}

A knee joint contracture, or the loss of passive range of motion (ROM) of the knee joint, affects normal mobility and negatively impacts a person's daily life. Contractures can severely inhibit a person's independence and healthy life style. They may require medical attention, especially when multiple limbs are affected. ${ }^{1}$

Contractures can develop in a variety of ailments, including congenital disorders, ${ }^{2}$ arthritis, ${ }^{3}$ secondary to injury, ${ }^{1}$ as a complication of arthroplasty, ${ }^{4}$ and after extended periods of immobility. ${ }^{5-7}$ In one report, $>70 \%$ of institutionalized elderly patients who were immobile had at least one joint contracture compared with none in mobile patients. ${ }^{6}$ Prolonged immobility is also enforced in the intensive care unit; a stay of over 2 weeks was associated with at least one functionally restrictive contracture in over a third of patients, ${ }^{5}$ and $70 \%$ of these patients still had self-reported issues with reduced mobility at 3.3 years after discharge. ${ }^{8}$ In knee arthroplasty patients, a 3.6\% occurrence of contractures was reported at 2 years post surgery ${ }^{4}$; in the US, this corresponds to 4,630 new cases of knee flexion contractures every year out of the 
130,000 knee replacement surgeries performed on patients with disabling arthritis. ${ }^{9}$

Joint contractures develop slowly, are chronic, and have been treated with limited success. ${ }^{10-12}$ While prevention is the preferred approach, most contractures are diagnosed only after they have become chronic. The most common treatment is physical therapy with manual stretching and splinting, even though their efficacy lacks evidence. ${ }^{1,11,13}$ Posterior knee joint capsule surgical release is considered for functionally limiting contractures refractory to medical management. While effective, the surgery is reported to be technically demanding, is risky due to important blood vessels and nerves in close proximity to the capsule, and can create instability and other surgical complications. ${ }^{11}$ The current treatments for joint contractures are inadequate, and knee joint contractures can permanently impair a person's mobility.

Pharmacological treatments for joint contractures are emerging and most are in the experimental phase. ${ }^{14,15}$ A treatment with collagenase purified from Clostridium histolyticum (Endo Pharmaceuticals Inc., Xiaflex ${ }^{\circledR}$, Malvern, PA, USA), further referred to as $\mathrm{CCH}$, has been approved for treatment of contractures in Dupuytren's disease and Peyronie's disease and is currently in commercial review for adhesive capsulitis in the shoulder. ${ }^{16,17}$ In Dupuytren's disease, a build-up of collagen in the palmar and plantar aponeuroses is thought to limit the mobility of joints in the fingers and toes, ${ }^{18}$ and therefore, collagenase treatment is a logical course. Regarding knee flexion contractures, a rat model has demonstrated fibrosis of the posterior capsule as a predominant cause limiting knee extension. ${ }^{11,18-21}$ Further research has demonstrated changes in gene expression of collagen pathways ${ }^{9}$ as well as changes in collagen protein levels and organization in the knee capsule during contracture development. ${ }^{3,18,22,23}$ We hypothesized that pharmacologically targeting the collagen matrix in the posterior capsule of a knee with flexion contracture would result in increased ROM in extension. As a proof of concept, collagenases were locally administered to treat immobilityinduced knee flexion contracture in the rat model. ${ }^{24}$

\section{Materials and methods}

\section{Animals and surgery}

The following was approved by the University of Ottawa's Animal Care Committee under the protocol name "Knee Joint Contractures: Reversibility and Molecular pathways" (protocol no ME-2461). Animal welfare guidelines from the University of Ottawa's Animal Care Committee were followed. Twenty adult male Sprague Dawley rats (Charles River Laboratories, St-Constant, Quebec, Canada) had one hind leg surgically immobilized at a $45^{\circ}$ flexion angle between the femur and tibia, as previously described, ${ }^{23}$ for 4 weeks. Briefly, under halothane anesthesia, steel screws were used to fix a Delrin ${ }^{\circledR}$ plate (DuPont Engineering Polymers, Wilmington, DE, USA) internally but extra-articularly to the proximal femur and distal tibia. The side of surgery was alternated between rats and the contralateral hind legs had no intervention. After 4 weeks of immobilization, screws and plates were removed under similar surgical conditions. $\mathrm{CCH}$ (Endo Pharmaceuticals Inc., Xiaflex, Malvern, PA, USA) was suspended in the provided saline-calcium buffer according to the manufacturer's guide. At the same time as plate removal, the experimental knees were intra-articularly injected with a sterile 27-gauge needle using a lateral approach. Treated rats received $50 \mu \mathrm{L}$ of $0.6 \mathrm{mg} / \mathrm{mL} \mathrm{CCH}$ and control rats received $50 \mu \mathrm{L}$ of the provided buffer. The dosage of collagenase selected was based on the manufacturer's recommendation of reconstitution volume of $0.25 \mathrm{~mL}$ to be added to the vial containing $0.58 \mathrm{mg}$ of dry collagenase. From the reconstituted collagenase, the injected volume was $50 \mu \mathrm{L}$ and corresponded to half of the previously reported intra-articular volume of the rat knee, that is, $100 \mu \mathrm{L} .{ }^{32}$ Rats were allowed 2 weeks of unassisted remobilization after plate removal and injection. ROM in extension was immediately measured after each rat was euthanized with carbon dioxide inhalation.

\section{ROM measurements}

ROM in extension of both the experimental and contralateral hind legs were measured using an automatic arthrometer developed by the Bone and Joint Research Laboratory at the University of Ottawa, as previously described. ${ }^{25}$ Briefly, the femur was clamped onto the arthrometer and a rotating arm approaching posteriorly at the ankle displaced the tibia at four predetermined torques: $2.5,7.5,12.5$, and $23.4 \mathrm{~N} \cdot \mathrm{cm}$. At each torque, a picture was captured with a mounted camera (Canon EOS-500D, Tokyo, Japan). The skin and posterior musculature at the calf and hamstrings were then sharply divided (ie, cut perpendicularly to the bone), and ROM measurements were repeated at the same four torques; this second set of data was referred to as "muscle off". Knee ROM measurements in extension were then conducted on the contralateral leg, with muscle on and muscle off. Images were imported into Image J software, ${ }^{26}$ and the angle between the femur and tibia was measured using landmarks of the arthrometer (femur clamp and center of rotation) and the lateral malleolus of the tibia. The angle was measured using ImageJ, and the $\mathrm{R}$ environment ${ }^{27}$ was used for statistical analysis. ${ }^{28}$ 


\section{Histology}

After mechanical testing with the arthrometer, rat knee joints were fixed in $10 \%$ formalin overnight at $4{ }^{\circ} \mathrm{C}$. Knees were decalcified in $10 \%$ ethylenediaminetetraacetic acid for 60 days. Knees were embedded in paraffin, and $7 \mu \mathrm{m}$ sagittal sections at the medial mid-condylar level were prepared. Primary antibodies for Col I (GeneTex, GTX20292, Irvine, CA, USA) and Col III (Bioss Inc., bs-0549R, Woburn, MA, USA) were diluted in water in the ratio 1:300 and 1:500, respectively. Sections were incubated overnight at $4^{\circ} \mathrm{C}$. Positive staining was revealed with MACH 4 HRPPolymer (Biocare Medical, Concord, CA, USA) and Liquid DAB Substrate Pack (BioGenex, HK103-5K, Fremont, CA, USA). Sections were counterstained with hematoxylin (Thermo Electron Corporation, Waltham, MA, USA) and mounted. Four fields along the posterior capsule in the stained sections were visualized with an Olympus BH-2 light microscope and a Marlin F080C digital camera (Allied Vision Technologies, Exton, PA, USA) with AVT Smartview 1.5.1 software at $66 \times$. Images were imported to Image $J$ and converted to an RGB stack. The blue channel was chosen to eliminate intensity from the hematoxylin staining and focus on the immunostain. The intensity of the staining was quantified using the area under the plot profile graph and a completely black image would be interpreted as $100 \%$ staining intensity. Staining intensity was measured in all knee specimens in fields viewed at $20 \times$ magnification. The number of fields analyzed from each group was as follows: $n=44$ fields from buffer knees, $n=36$ fields from collagenase-treated knees, and $n=64$ fields from contralateral knees. Results were reported as the average \pm SD. An unpaired Student's $t$-test was used to test for differences between $\mathrm{CCH}$-injected, buffer-injected, and contralateral knee capsule staining.

Other sections of $\mathrm{CCH}$ - and buffer-injected knees were stained with hematoxylin and eosin to inspect for articular damage to the femoral and tibial articular cartilage. Representative slides at $13.2 \times$ and $33 \times$ are presented.

\section{Results Knee ROM in extension}

Pictures of the automated arthrometer with a rat leg positioned for ROM measurement of knee extension are shown in Figure 1. The rat femur was positioned over the center of rotation of the arthrometer and the leg rested on the movable arm that was pushed in extension at $0.69 \mathrm{rad} / \mathrm{second}$ to reach the following torques: $2.5 \mathrm{~N} \cdot \mathrm{cm}$ (Torque 1), $7.5 \mathrm{~N} \cdot \mathrm{cm}$ (Torque 2), and $12.5 \mathrm{~N} \cdot \mathrm{cm}$ (Torque 3 ). Measures of ROM in extension for each torque are indicated. ROM averages for immobilized and contralateral knees of each experimental group (CCH-treated and buffer-treated rats) are presented in Table 1 and Figure 2. The contralateral leg was $+37.7^{\circ} \pm 1.2^{\circ}$ more extensible than the immobilized leg $(p<0.001$, Table 2$)$, and ROM was significantly higher $\left(+33.8^{\circ} \pm 1.7^{\circ}\right)$ as the torque increased from 2.5 to $23.4 \mathrm{~N} \cdot \mathrm{cm}(p<0.001$, Table 2$)$.
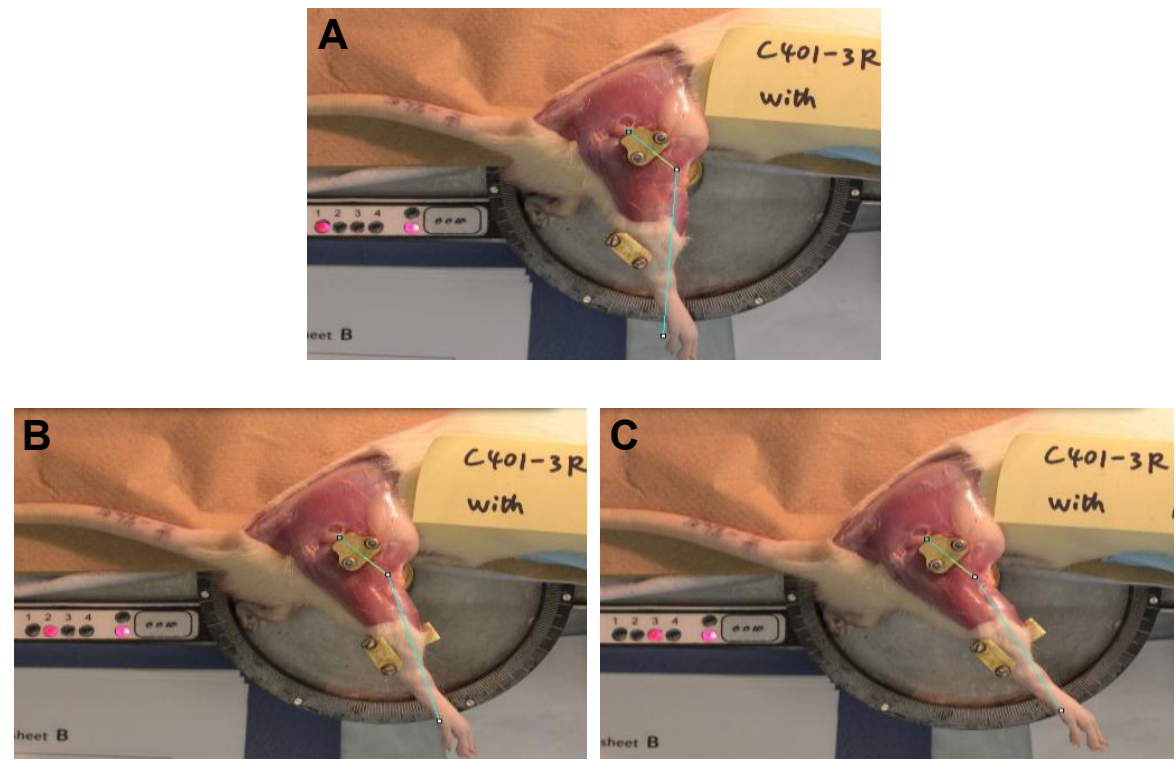

Figure I Pictures of the automatic arthrometer developed by The Bone and Joint Laboratory and used to measure the knee ROM in extension. Notes: Images show a rat leg without skin and with muscle mounted on the arthrometer with measures of the ROM in extension at $(\mathbf{A})$ torque $I\left(I 3 I^{\circ}\right)$, $(B)$ torque $2\left(I 49^{\circ}\right)$, and $(\mathbf{C})$ torque $3\left(161^{\circ}\right)$.

Abbreviation: ROM, range of motion. 
Table I Mean extension angles between tibia and femur for both legs of $\mathrm{CCH}$-injected and buffer-injected rats, with muscle on and muscle off and at four torques

\begin{tabular}{|c|c|c|c|c|c|c|c|c|c|}
\hline \multirow[t]{3}{*}{ Muscle } & \multirow{3}{*}{$\begin{array}{l}\text { Torque } \\
(\mathbf{N} \cdot \mathrm{cm})\end{array}$} & \multicolumn{4}{|c|}{$\mathrm{CCH}(n=9)$} & \multicolumn{4}{|c|}{ Buffer $(n=I I)$} \\
\hline & & \multicolumn{2}{|c|}{ Immobilized } & \multicolumn{2}{|c|}{ Contralateral } & \multicolumn{2}{|c|}{ Immobilized } & \multicolumn{2}{|c|}{ Contralateral } \\
\hline & & Mean $\left({ }^{\circ}\right)$ & SEM & Mean $\left({ }^{\circ}\right)$ & SEM & Mean $\left({ }^{\circ}\right)$ & SEM & Mean $\left({ }^{\circ}\right)$ & SEM \\
\hline \multirow[t]{4}{*}{ On } & 2.5 & 93.3 & 6.7 & 134.7 & 4.5 & 89.5 & 2.8 & $|25|$. & 2.7 \\
\hline & 7.5 & 98.4 & 7.2 & 143.8 & 4.3 & 95.7 & 3.2 & 134.5 & 2.6 \\
\hline & 12.5 & 116.2 & 7.7 & 156.3 & 3.0 & 105.6 & 3.7 & 145.4 & 2.4 \\
\hline & 23.4 & 133.4 & 6.4 & 169.4 & 2.6 & 119.1 & 4.1 & 157.0 & 2.3 \\
\hline \multirow[t]{4}{*}{ Off } & 2.5 & 128.5 & 6.4 & 156.0 & 2.1 & 111.8 & 8.8 & 147.2 & 2.3 \\
\hline & 7.5 & 134.8 & 6.8 & 162.0 & 2.1 & 117.7 & 2.6 & 153.2 & 2.2 \\
\hline & 12.5 & 142.6 & 7.6 & 173.6 & 2.0 & 126.8 & 2.7 & 161.8 & 2.5 \\
\hline & 23.4 & 175.5 & 9.3 & 189.6 & 5.4 & 140.5 & 4.2 & 173.7 & 3.6 \\
\hline
\end{tabular}

Notes: After 4 weeks of immobilization, experimental knees were intra-articularly injected with CCH $(50 \mu \mathrm{L}, 0.6 \mu \mathrm{g} / \mu \mathrm{L})$ or buffer $(50 \mu \mathrm{L})$. After 2 weeks of spontaneous remobilization, ROM was measured with an automatic arthrometer at torques $2.5,7.5,12.5$, and $23.4 \mathrm{~N} \cdot \mathrm{cm}$, with muscle on and muscle off. Mean extension angles are presented with standard error. (Statistical analysis is provided in Table 2.)

Abbreviations: $\mathrm{CCH}$, collagenase Clostridium histolyticum; ROM, range of motion; SEM, standard error of mean.

For "muscle on" data, ROM was $+23.8^{\circ} \pm 1.2^{\circ}$ compared to "muscle off" ( $p<0.001$, Tables 1 and 2$)$. The ROM of immobilized knees injected with $\mathrm{CCH}$ was $+8.0^{\circ} \pm 3.8^{\circ}$ compared to buffer injected ( $p<0.05$, Table 2$)$. Variability of ROM measures in each group is displayed by box plots (Figure 3). A higher variability is evident in $\mathrm{CCH}$-injected knees compared with buffer-injected knees; ROM data for buffer-injected knees distributed closely around the median compared with CCH-injected knees (Figure 3).

\section{Histology}

Col I staining was significantly higher in the posterior capsule of experimental knees than in the contralateral knees
(Figure 4A, $p<0.01$ for $\mathrm{CCH}$ injected and $p=0.05$ for buffer injected). The increase in Col I staining in the posterior capsule of $\mathrm{CCH}$-injected knees compared to buffer-treated knees did not reach statistical significance $(p>0.05)$. Representative images of stained knee sections used for the quantitative analysis are presented in Figure 4B. There were significant increases in Col III staining of CCH-treated capsules compared with both buffer-treated $(p<0.01)$ and contralateral $(p<0.01)$ samples.

The surfaces of the articular cartilage of the femur and tibia of contralateral knee joints appeared smooth and undamaged (Figure 5). The articular cartilage of both buffer-injected and $\mathrm{CCH}$-injected knees presented some

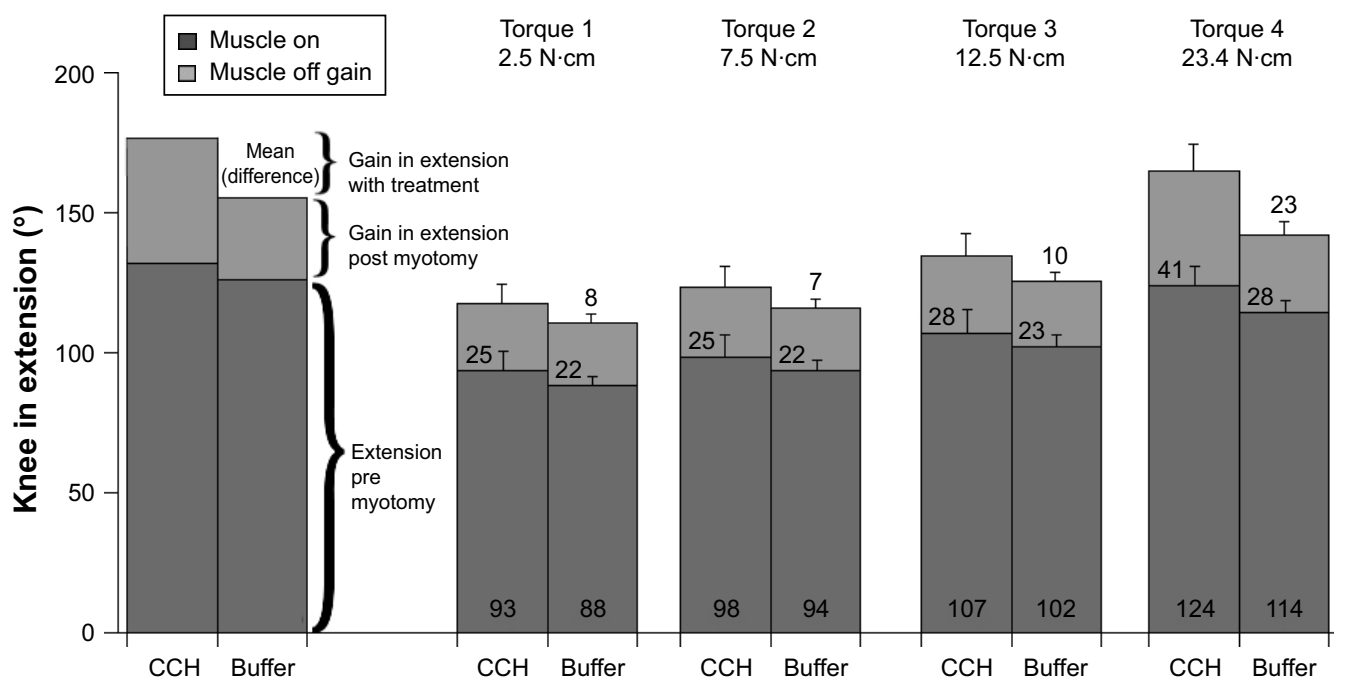

Figure 2 Mean extension angles of $\mathrm{CCH}$-injected and buffer-injected experimental rat knees.

Notes: After 4 weeks of immobilization, experimental knees were intra-articularly injected and given 2 weeks for spontaneous remobilization. Mean angles were calculated after measurement of ROM at four torques $(2.5,7.5,12.5$, and $23.4 \mathrm{~N} \cdot \mathrm{cm}$ ) with muscle on and then after sectioning the muscle (muscle off). Mean ROM values are given within each bar along with the mean gain in ROM with $\mathrm{CCH}$ treatment (number above buffer bar). The difference between the groups was most noticeable with muscle off and at the highest torque, where ROM in the treated legs was higher than the control legs. Means are presented with standard error. Collagenase, $\mathrm{n}=9$; buffer, $\mathrm{n}=\mathrm{I} \mathrm{I}$. (Statistical analysis is presented in Table 2.)

Abbreviations: $\mathrm{CCH}$, collagenase Clostridium histolyticum; ROM, range of motion. 
Table 2 Statistical linear mixed effect modeling to determine the effect of $\mathrm{CCH}$ injection compared to buffer injection on the ROM of a rat knee with contracture

\begin{tabular}{|c|c|c|c|c|c|}
\hline $\begin{array}{l}\text { Linear mod } \\
\text { (error: rat.I }\end{array}$ & $\begin{array}{l}\text { el: ROM } \\
\text { D) }\end{array}$ & injecti & torque + & cle + & \\
\hline $\begin{array}{l}\text { Estimated } \\
\text { increase }\left({ }^{\circ}\right)\end{array}$ & $\begin{array}{l}\text { Standard } \\
\text { error }\end{array}$ & $p$-value & $\begin{array}{l}\text { Estimated } \\
\text { increase }\left({ }^{\circ}\right)\end{array}$ & $\begin{array}{l}\text { Standard } \\
\text { error }\end{array}$ & $p$-value \\
\hline Buffer to $\mathrm{C}$ & $\mathrm{CH}$ & & $\begin{array}{l}\text { Torque } 2.5 \\
23.4 \mathrm{~N} \cdot \mathrm{cm}\end{array}$ & $\mathrm{N} \cdot \mathrm{cm}$ to to & rque \\
\hline 8.0 & 3.8 & 0.046 & 33.8 & 1.7 & $1.0 \times 10^{-58}$ \\
\hline Muscle on $t$ & o muscle o & & Immobilized & d to contra & lateral \\
\hline 23.8 & 1.2 & $3.9 \times 10^{-55}$ & 37.7 & 1.2 & $4.3 \times 10^{-95}$ \\
\hline
\end{tabular}

Notes: $\mathrm{CCH}$ injection with 2 weeks of spontaneous remobilization increases ROM by $8.0^{\circ}\left( \pm 3.8^{\circ}\right)$ with a $p$-value of $0.046 . \mathrm{CCH}, \mathrm{n}=9$; buffer, $\mathrm{n}=\mathrm{II}$.

Abbreviations: $\mathrm{CCH}$, collagenase Clostridium histolyticum; ROM, range of motion.

irregularities limited to the surface and was comparable for both experimental groups.

\section{Discussion}

This study sought to evaluate the potential therapeutic effect of a single intra-articular collagenase injection to reverse knee flexion contractures. Supporting our hypothesis, rat knees with flexion contractures gained $8.0^{\circ} \pm 3.8^{\circ}$ in extension; a statistically significant increase in ROM compared with buffer-injected knees. In clinical terms, a gain of $8.0^{\circ} \pm 3.8^{\circ}$ of extension at the knee can improve a person's gait, energy expenditure, and function.

The tissues targeted by the intra-articular injection of $\mathrm{CCH}$ and the molecular mechanisms involved were investigated. ROM measured after sectioning transarticular muscles confirmed the involvement of the posterior capsule in preventing full extension of the knee. In agreement with previous literature, our data indicate that joint contractures induced by immobilization are caused by myogenic and arthrogenic structures, mainly the posterior articular capsule. ${ }^{25}$ Moreover, for long-lasting contractures, the capsule contribution to the contracture was more important than the muscular contribution..$^{29}$ After 4 weeks of immobilization, spontaneous recovery of ROM was limited, and joint contractures persisted for at least 16 weeks after remobilization. ${ }^{29,30}$ At 4 weeks of immobilization with no remobilization, a previous study showed flexion contractures with a mean of $101^{\circ}$ in extension. ${ }^{30}$ In the current study, a 4-week contracture was treated with $\mathrm{CCH}$, and its effect was measured after 2 weeks. The experimental protocol tested the effects of $\mathrm{CCH}$ on established contractures that do not reverse with spontaneous remobilization.

The increase in rat knee range of extension after treatment was significant; however, full extension was not restored. The incomplete resolution of the contracture after a single injection of intra-articular collagenase may be due to dosing, frequency, and/or timing. Dosing and frequency in this study were based on previous literature and the clinical monograph of $\mathrm{CCH}$ for the treatment of Dupuytren's contractures. ${ }^{31}$ A single injection of $0.58 \mathrm{mg}$ of $\mathrm{CCH}$ is recommended to restore finger joint mobility restricted by the fascia in the palm of the hand. ${ }^{31}$ For our study, the authors chose to mimic dosing as is prescribed to the patients of Dupuytren's contracture. Further studies may adjust and modify dosing to determine the most optimal posology. Previous studies have shown that a maximum of $100 \mu \mathrm{L}$ can be injected into a rat

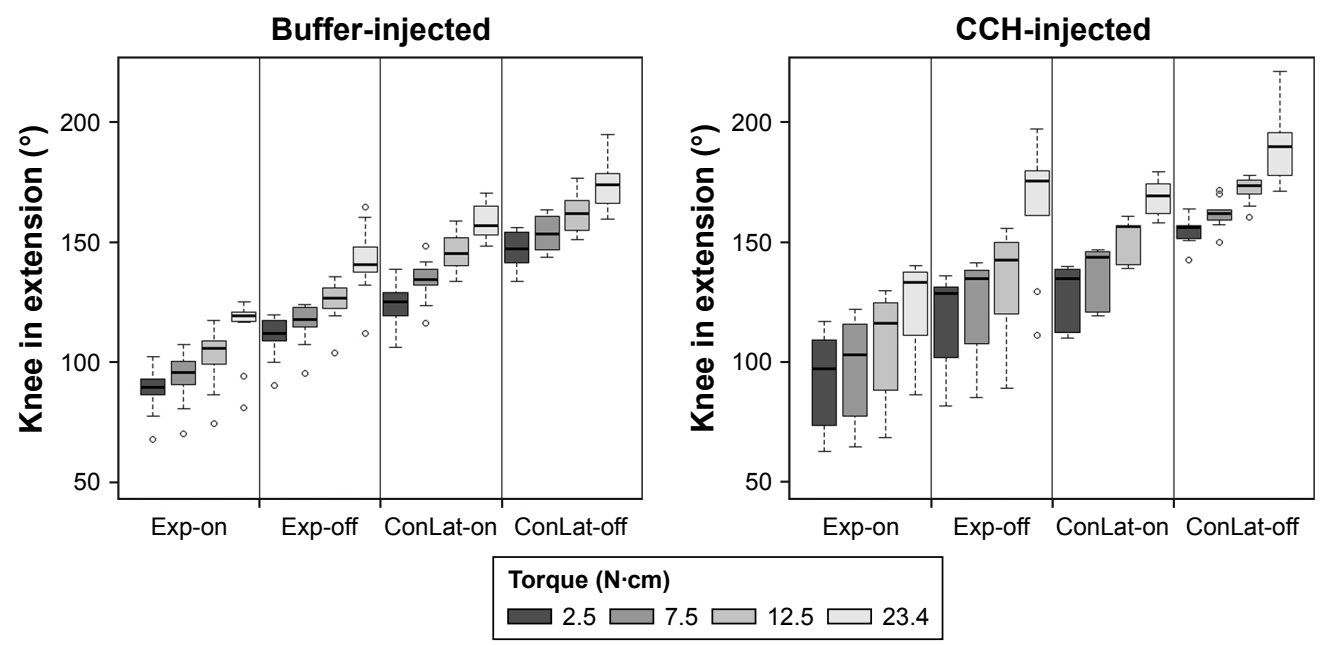

Figure 3 Box plots showing the degree of variation for ROM for experimental and contralateral knees of buffer-injected and $\mathrm{CCH}$-injected rats.

Notes: The black lines represent median values for each group: experimental (Exp) or contralateral (ConLat) with muscle on (on) or sectioned (off). Each box represents the interquartile range, whiskers indicate the range of highest and lowest extension angles that are nonoutliers, and outliers (outside I.5 times the interquartile range) are plotted as individual points. $\mathrm{CCH}, \mathrm{n}=9$; buffer, $\mathrm{n}=\mathrm{II}$.

Abbreviations: $\mathrm{CCH}$, collagenase Clostridium histolyticum; ROM, range of motion. 

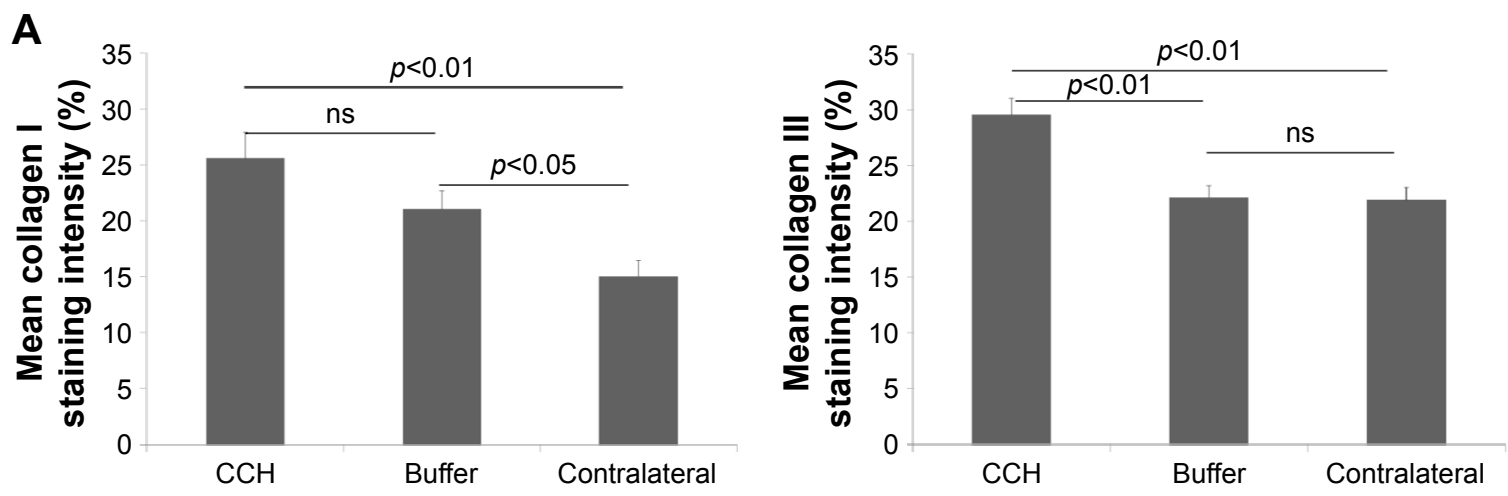

B

Buffer-injected col I

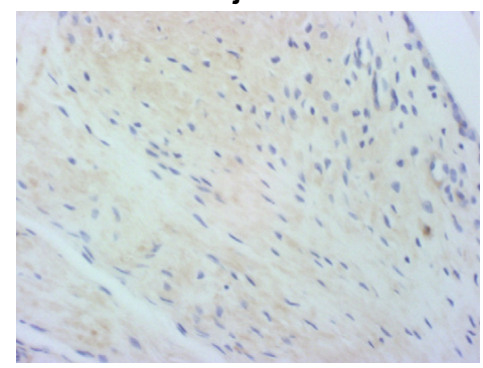

Buffer-injected col III

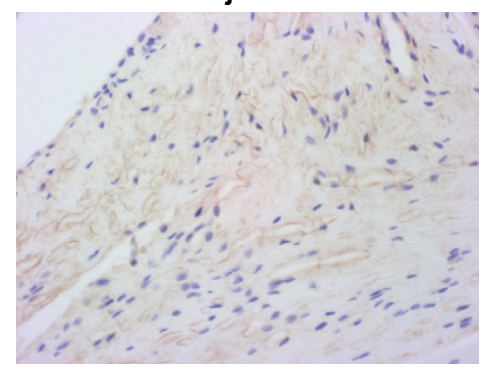

CCH-injected col I

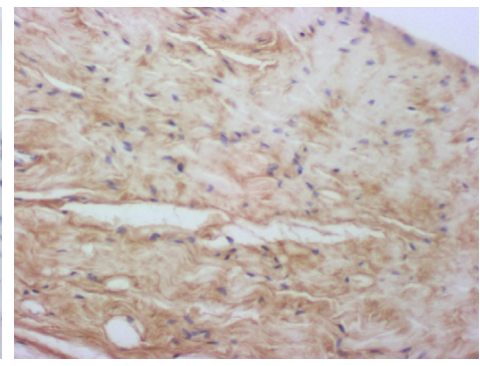

CCH-injected col III

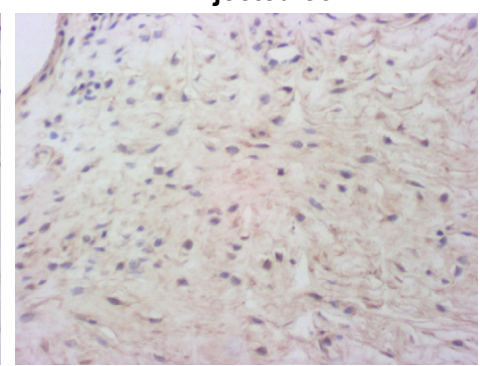

Figure 4 Immunostaining of collagen type I and collagen type III in the posterior capsule from contralateral knees and experimental knees intra-articularly injected with $\mathrm{CCH}$ or buffer.

Notes: (A) The extracellular matrix staining was quantified using Imagej. Intensity of staining as a percentage is presented in the bar graphs (with total black considered as 100\%). Experimental capsules (both CCH and buffer) showed significantly more staining than contralateral for collagen type I. Quantification of collagen type III staining for the same capsules showed a significant increase in staining in the $\mathrm{CCH}$-treated capsule compared with buffer-treated and contralateral capsules ( $\mathrm{n}=44$ fields from buffer knees, $\mathrm{n}=36$ fields from $\mathrm{CCH}$ knees, and $\mathrm{n}=64$ fields from contralateral knees). (B) Representative micrographs of sections from the posterior knee capsule from joint injected with either buffer or collagenase $(\mathrm{CCH})$ and stained with collagen type I or type III are shown.

Abbreviation: ns, not significant.

knee joint without disruption, ${ }^{32}$ and $50 \mu \mathrm{L}$ volumes have been used in other intra-articular rat knee injection studies. ${ }^{33-35}$ Similar to Dupuytren's treatment, $\mathrm{CCH}$ in our model was administered after the contracture was established. Proceeding from this study, the optimization of posology, timing, and frequency of administration is necessary to address residual contractures. Current treatment of Dupuytren's contractures includes finger stretching in extension performed at 24-72 hours after injection to facilitate disruption of collagen build-up. ${ }^{36}$ Also, $\mathrm{CCH}$ injections may be repeated up to three times at $\sim 4$-week intervals. Similarly, for knee flexion contracture, establishment of a collagenase treatment to increase ROM could include physical therapy and stretching, more than one injection, and/or a higher dosage.
The amplitude of ROM gained after CCH injection was comparable to previously published studies designed to test the effects of injected intra-articular drug compounds on contractures using animal models. In a study with experimental rats undergoing surgical knee trauma with postoperative immobilization, a single intra-articular injection of montelukast (leukotriene receptor antagonist), triamcinolone (corticosteroid), and forskolin (increases cAMP) led to increases in extension of $12^{\circ}, 23^{\circ}$, and $10^{\circ}$, respectively, compared with control rats. ${ }^{37}$ In a mouse model of immobilized knees, intra-articular injection of oligonucleotides designed to inhibit the expression of the hypoxia inducible factor 1 gene gained $\sim 10^{\circ}$ extension at 1 week after injection. ${ }^{38}$ Similar to intra-articular-injected $\mathrm{CCH}$, these compounds increased 


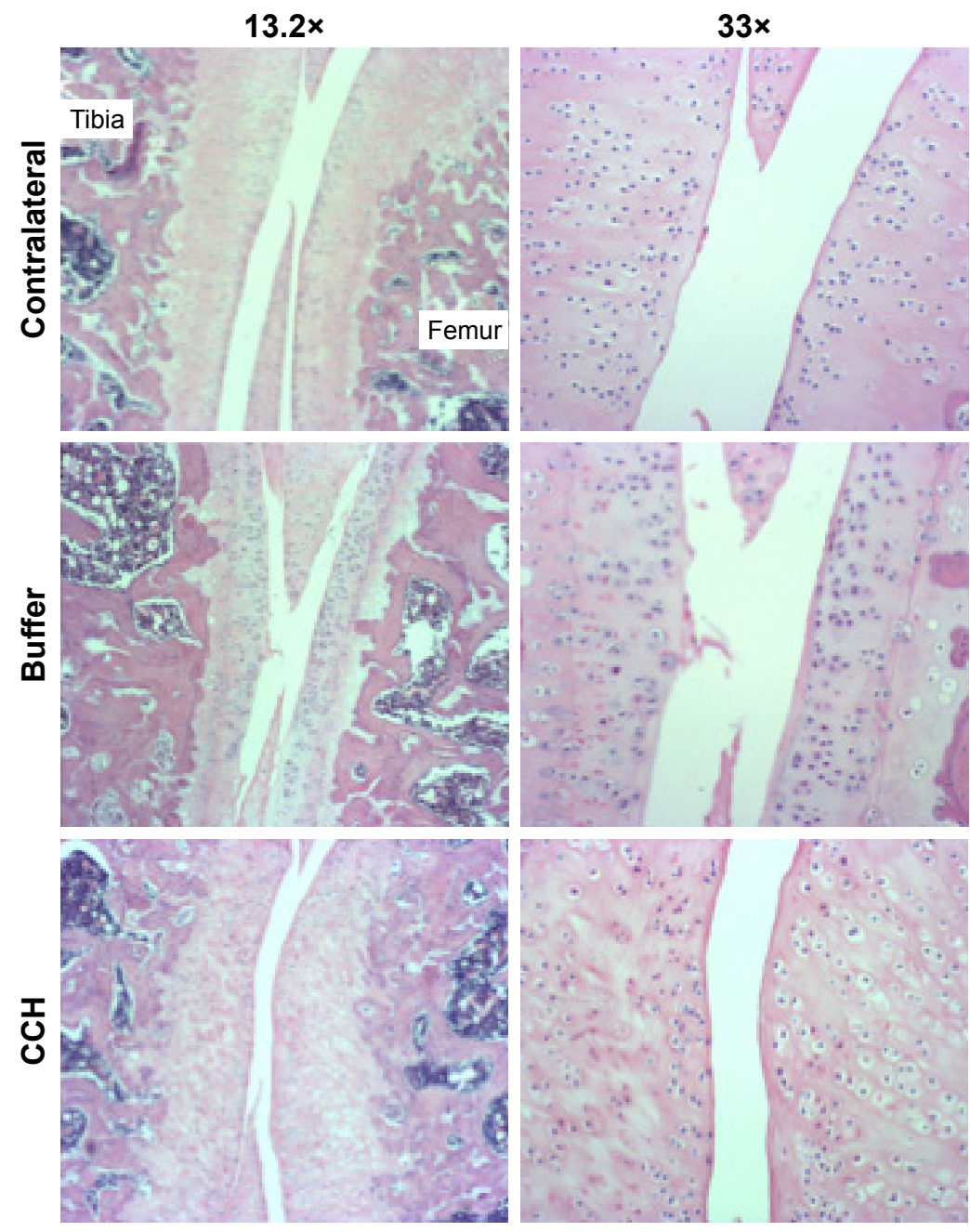

Figure 5 Hematoxylin and eosin staining of contralateral knees and experimental knees intra-articularly injected with $\mathrm{CCH}$ or buffer. Notes: Original magnification of $13.2 \times$ and $33 \times$. The articular cartilage along the femoral condyles and tibial plateaus of the contralateral joints is smooth and undamaged. The cartilage of joints with contractures is uneven; however, $\mathrm{CCH}$-injected knee joints show no further damage compared to the buffer-injected joints.

Abbreviation: $\mathrm{CCH}$, collagenase Clostridium histolyticum.

ROM but did not completely reverse the contracture. While rat knee ROM cannot be directly correlated with human knee ROM, two studies report the benefit of even a slight gain in knee extension. In the first study, a loss of knee extension as little as $5^{\circ}$ resulted in a limp and abnormal gait. ${ }^{11}$ In the second study of 18 patients who had received arthroscopic lysis of posterior capsule adhesions, a mean improvement of $6.11^{\circ}$ was reported between preoperative and postoperative ROM flexion contracture. ${ }^{39}$ Thirteen of the 15 patients the researchers were able to contact indicated overall satisfaction with the procedure..$^{39}$ These two studies support not only a statistical but also a functional impact of a gain of $8^{\circ}$ of knee extension.

The molecular mechanisms involved in capsule changes leading to joint contractures are not well understood. Abnormal fibrosis involving the extracellular matrix, in particular collagen content and organization, has been proposed. Strategies to target articular fibrosis and restore complete ROM have been tested with limited success. Intra-articular injection of decorin had no significant effect on ROM in a rabbit knee contracture model. ${ }^{40,41}$ Collagenase therapy has the potential to treat a variety of conditions caused by excess collagen and reorganization of the matrix in connective tissues. CCH for the treatment of Dupuytren's hand contractures has had its enzyme activity of degrading collagen extensively studied..$^{42}$ Prokaryotic collagenase, such as those from C. histolyticum, has broad substrate specificity and has been reported to degrade a wide variety of collagen types. ${ }^{43,44}$ The mix of purified collagenase used in the current study has been shown to spare collagen type IV, ${ }^{42}$ the main component of basement membrane. This has important clinical value given the proximity of neurovascular structures to the posterior 
knee capsule. The current study demonstrates a potential new use of $\mathrm{CCH}$ to treat knee flexion contractures.

A concern with using intra-articular collagenase treatments for joint contractures is the possible degradation of collagen type II in the articular cartilage. In a rabbit model, knee injections of $C$. histolyticum collagenase have been used to induce osteoarthritis (OA) ${ }^{45}$ However, the dosage used to induce OA was more than 30 -fold higher (1 mg compared to $30 \mu \mathrm{g}$ ), and the injections were performed twice, at days 1 and 4, compared to our experiment where injection was performed only once. ${ }^{45}$ Contractures secondary to immobilization without collagenase injections are known to deteriorate the articular cartilage, including an increase in surface irregularity. ${ }^{46}$ Microscopic examination of rat knees with contracture showed surface irregularity compared with the contralateral knees, likely resulted from the immobilization, as previously reported. ${ }^{46}$ Qualitatively, knees injected with $\mathrm{CCH}$ showed no further structural damage to the cartilage compared to the buffer-injected knees. The experimental knees were examined after only 2 weeks of remobilization and it is possible that there would be further damage after 2 weeks post injection. The stability and long-term activity of this collagenase mix have not yet been fully characterized. Clinically, patients with preoperative contractures have an increased likelihood of developing contracture post total knee arthroplasty. ${ }^{4}$ In these cases, the entire cartilage surfaces are replaced with material components, and there is no articular cartilage available to be subjected to damage from collagenase. This constitutes a potential application of intra-articular collagenase treatment of joint contractures in patients who do not have viable cartilage. Potential damage of other connective tissues present in the knee, such as tendons and ligaments, has not yet been investigated.

An interesting finding in this study is the significantly higher ROM in the contralateral knees of the rats treated with $\mathrm{CCH}$ compared with those of the buffer-treated rats, even though no interventions were performed on the contralateral legs. This finding is consistent with data from a rabbit contracture model showing additional contractures developing in contralateral knee of immobilized animals compared with unoperated controls..$^{14}$ These results are also congruent with clinical studies of OA patients with knee flexion contractures who also had limited ROM in the contralateral leg when compared with OA patients without contractures. ${ }^{47}$ Contralateral legs may compensate for the lack in ROM in the affected leg by establishing habitual flexion. In this experiment, we conclude that the increased ROM in the treated knee allowed for further ROM in the contralateral leg, supporting the evidence that the treatment increases functional ROM.
Limitations to this study include two rats in the $\mathrm{CCH}$ treated group that had notably increased the variability of ROM and were identified as outliers at the highest torque measured post myotomy. Their lower ROM could be attributed to the blind injection missing the intra-articular space, and $\mathrm{CCH}$ may not have reached the posterior capsule. Training for intra-articular injection accuracy was performed on Sprague-Dawley rats that had been euthanized for other studies using Evan's blue dye. A lateral approach with the knee in flexion performed best. While the blind injection conformed to the overall goal of minimal interference with the knee joint, it is possible that, despite training, some injections missed the rat knee intra-articular space. Future studies with radiologic support may improve the yield of successful drug delivery. This study was limited to 4 weeks of knee immobilization with a 2-week follow-up. The effect of $\mathrm{CCH}$ on contractures developed from longer durations of immobilization was not studied. Given the increase in ROM after sectioning of the muscles, a myogenic component of the contracture is also present. This myogenic component must also be recognized in order to efficiently treat the loss of ROM.

\section{Conclusion}

Our study adds to the preclinical research for the use of injectable prokaryotic collagenase as a treatment for a variety of collagen-related diseases. Research investigating the potential of administering $\mathrm{CCH}$ is underway, including clinical trials for frozen shoulder syndrome (adhesive capsulitis), lipoma, and uterine fibroids. ${ }^{16} \mathrm{CCH}$ injections have so far been successful in treating Dupuytren's and Peyronie's diseases. ${ }^{48,49}$ Further research should aim to examine the optimal use of collagenase in treating joint contractures.

\section{Acknowledgments}

This study was supported in part by Canadian Institutes of Health Research Operating Grant MOP 97831. We thank Winnie Nie for participating in ROM measurements and histology slide preparation, Eileen Franklin and Kim Yates for assistance during surgeries, and Dr Ana Giassi and the Histology Core Laboratory of the University of Ottawa for tissue processing.

\section{Disclosure}

The authors report no conflicts of interest in this work.

\section{References}

1. Campbell M, Dudek N, Trudel G. Joint contractures. In: Frontera WR, Silver JK, Rizzo TD, editors. Essentials of Physical Medicine and Rehabilitation: Musculoskeletal Disorders, Pain, and Rehabilitation. Philadelphia, PA: Elsevier Saunders; 2014:651-655. 
2. Hall JG. Arthrogryposis (multiple congenital contractures): diagnostic approach to etiology, classification, genetics, and general principles. Eur J Med Genet. 2014;57(8):464-472.

3. Campbell TM, Trudel G, Wong KK, Laneuville O. Genome wide gene expression analysis of the posterior capsule in patients with osteoarthritis and knee flexion contracture. J Rheumatol. 2014;41(11):2232-2239.

4. Ritter MA, Lutgring JD, Davis KE, Berend ME, Pierson JL, Meneghini RM. The role of flexion contracture on outcomes in primary total knee arthroplasty. J Arthroplasty. 2007;22(8):1092-1096.

5. Clavet H, Hebert PC, Fergusson D, Doucette S, Trudel G. Joint contracture following prolonged stay in the intensive care unit. CMAJ. 2008; 178(6):691-697.

6. Fergusson D, Hutton B, Drodge A. The epidemiology of major joint contractures: a systematic review of the literature. Clin Orthop Relat Res. 2006;456:22-29.

7. Selikson S, Damus K, Hamerman D. Risk factors associated with immobility. J Am Geriatr Soc. 1988;36(8):707-712.

8. Clavet H, Doucette S, Trudel G. Joint contractures in the intensive care unit: quality of life and function 3.3 years after hospital discharge. Disabil Rehabil. 2014;37(3):207-213.

9. Wong K, Sun F, Trudel G, Sebastiani P, Laneuville O. Temporal gene expression profiling of the rat knee joint capsule during immobilizationinduced joint contractures. BMC Musculoskelet Disord. 2015;16:125.

10. Trudel G, Laneuville O, Uhthoff HK. Joint contractures. Clin Orthop Relat Res. 2007;456(2).

11. Chen MR, Dragoo JL. Arthroscopic releases for arthrofibrosis of the knee. J Am Acad Orthop Surg. 2011;19(11):709-716.

12. Prabhu RK, Swaminathan N, Harvey LA. Passive movements for the treatment and prevention of contractures (Review). Cochrane Database Syst Rev. 2013;12:CD009331.

13. Harvey LA, Katalinic OM, Herbert RD, Moseley AM, Lannin NA, Schurr K. Stretch for the treatment and prevention of contractures. Cochrane Database Syst Rev. 2017;1:CD007455.

14. Abdel MP, Morrey ME, Grill DE, et al. Effects of joint contracture on the contralateral unoperated limb in a rabbit knee contracture model: a biomechanical and genetic study. J Orthop Res. 2012;30(10): 1581-1585.

15. Park KD, Nam HS, Lee JK, Kim YJ, Park Y. Treatment effects of ultrasound-guided capsular distension with hyaluronic acid in adhesive capsulitis of the shoulder. Arch Phys Med Rehabil. 2013;94(2):264-270.

16. Biospecifics Technology Corp. Biospecifics 2017 Corporate Presentation. Biospecifics Technology. [Online cited June 2017]. Available from: http://biospecifics.com/images/stories/pdfs/bstccorporatepresentationjune2017.pdf. Accessed June 15, 2017.

17. Thurston AJ. Dupuytren's disease. J Bone Joint Surg Br. 2003;85(4): 469-477.

18. Lee S, Sakurai T, Ohsako M, Saura R, Hatta H, Atomi Y. Tissue stiffness induced by prolonged immobilization of the rat knee joint and relevance of AGEs (pentosidine). Connect Tissue Res. 2010;51(6): 467-477

19. Chimoto E, Hagiwara Y, Ando A, Itoi E. Progression of an arthrogenic motion restriction after immobilization in a rat experimental knee model. Ups J Med Sci. 2007;112(3):347-355.

20. Trudel G, Zhou J, Uhthoff HK, Laneuville O. Four weeks of mobility after 8 weeks of immobility fails to restore normal motion. Clin Orthop Relat Res. 2008;466(5):1239-1244.

21. Kleftogiannis F, Handley CJ, Campbell MA. Characterization of extracellular matrix macromolecules from bovine synovial capsule. J Orthop Res. 1994;12(3):365-374.

22. Matsumoto F, Trudel G, Uhthoff HK. High collagen type I and low collagen type III levels in knee joint contracture. Acta Orthop Scand. 2002;73(3):335-343

23. Hagiwara Y, Chimoto E, Ando A, Sasano Y, Hatori K, Itoi E. Expression of type I collagen in the capsule of a contracture knee in a rat model. Ups J Med Sci. 2007;112(3):356-365.

24. Trudel G, Uhthoff HK, Brown M. Extent and direction of joint motion limitation after prolonged immobility: an experimental study in the rat Arch Phys Med Rehabil. 1999;80(12):1542-1547.
25. Trudel G, Laneuville O, Coletta E, Goudreau L, Uhthoff HK. Quantitative and temporal differential recovery of articular and muscular limitations of knee joint contractures: results in a rat model. $J$ Appl Physiol (1985). 2014;117(7):730-737.

26. Schneider CA, Rasband WS, Eliceiri KW. NIH Image to ImageJ: 25 years of image analysis. Nat Methods. 2012;9(7):671-675.

27. R Core Team. R: a language and environment for statistical computing. [Online]. Available from: http://www.R-project.org/. Accessed June 15,2015 .

28. Bates D, Maechler M, Bolker B, Walker S. lme4: linear mixed-effects models using Eigen and S4, c2014. Available from: http:/CRAN.Rproject.org/package=lme4. Accessed August 13, 2015.

29. Trudel G, Uhthoff HK. Contractures secondary to immobility: is the restriction articular or muscular? An experimental longitudinal study in the rat knee. Arch Phys Med Rehabil. 2000;81(1):6-13.

30. Trudel G, Uhthoff HK, Goudreau L, Laneuville O. Quantitative analysis of the reversibility of knee flexion contractures with time: an experimental study using the rat model. BMC Musculoskelet Disord. 2014; $15: 338$.

31. Gilpin D, Coleman S, Hall S, Houston A, Karrasch J, Jones N. Injectable collagenase Clostridium histolyticum: a new nonsurgical treatment for Dupuytren's disease. J Hand Surg Am. 2010;35(12):2027.e1-2038.e1.

32. Riggin CN, Tucker JJ, Soslowsky LJ, Kuntz AF. Intra-articular tibiofemoral injection of a nonsteroidal anti-inflammatory drug has no detrimental effects on joint mechanics in a rat model. J Orthop Res. 2014; 32(11):1512-1519.

33. Bove SE, Calcaterra SL, Brooker RM, et al. Weight bearing as a measure of disease progression and efficacy of anti-inflammatory compounds in a model of monosodium iodoacetate-induced osteoarthritis. Osteoarthritis Cartilage. 2003;11(11):821-830.

34. Ashraf S, Mapp PI, Burston J, Bennett AJ, Chapman V, Walsh DA. Augmented pain behavioural responses to intra-articular injection of nerve growth factor in two animal models of osteoarthritis. Ann Rheum Dis. 2014;73(9):1710-1718.

35. Bajpayee AG, Scheu M, Grodzinsky AJ, Porter RM. Electrostatic interactions enable rapid penetration enhanced uptake and retention of intra-articular injected avidin in rat knee joints. J Orthop Res. 2014; 32(8):1044-1051

36. Warwick D, Arandes-Renú JM, Pajardi G, Witthaut J, Hurst LC. Collagenase Clostridium histolyticum: emerging practice patterns and treatment advances. J Plast Surg Hand Surg. 2016;50(5):251-261.

37. Efird W, Kellam P, Yeazell S, Weinhold P, Dahners LE. An evaluation of prophylactic treatments to prevent post traumatic joint stiffness. J Orthop Res. 2014;32(11):1520-1524.

38. Sotobayashi D, Kawahata H, Anada N, Ogihara T, Morishita R, Aoki M. Therapeutic effect of intra-articular injection of ribbon-type decoy oligonucleotides for hypoxia inducible factor-1 on joint contracture in an immobilized knee animal model. J Gene Med. 2016;18(8):180-192.

39. Bodendorfer BM, Kotler JA, Zelenty WD, Termanini K, Sanchez R, Argintar EH. Outcomes and predictors of success for arthroscopic lysis of adhesions for the stiff total knee arthroplasty. Orthopedics. 2017; 40(6):e1062-e1068.

40. Abdel MP, Morrey ME, Barlow JD, et al. Intra-articular decorin influences the fibrosis genetic expression profile in a rabbit model of joint contracture. Bone Joint Res. 2014;3(3):82-88.

41. Fukui N, Nakajima K, Tashiro T, Oda H, Nakamura K. Neutralization of fibroblast growth factor-2 reduces intra-articular adhesions. Clin Orthop. 2001;383:250-258.

42. Syed F, Thomas AN, Singh S, Kolluru V, Emeigh Hart SG, Bayat A. In vitro study of novel collagenase (XIAFLEX(R)) on dupuytren's disease fibroblasts displays unique drug related properties. PLoS One. 2012;7(2):e31430.

43. Eckhard U, Huesgen PF, Brandsetter H, Overall CM. Proteomic protease specificity profiling of clostridial collagenase reveals their intrinsic nature as dedicated degraders of collagen. J Proteomics. 2014;100:102-114.

44. Eckhard U, Schonauer E, Ducka P, Briza P, Nuss D, Brandsetter H. Biochemical characterization of the catalytic domains of three different clostridial collagenase. Biol Chem. 2009;390(1):11-18. 
45. Kikuchi T, Sakuta T, Yamaguchi T. Intra-articular injection of collagenase induces experimental osteoarthritis in mature rabbits. Osteoarthritis Cartilage. 1998;6(3):177-186.

46. Trudel G, Himori K, Goudreau L, Uhthoff HK. Measurement of articular cartilage surface irregularity in rat knee contracture. J Rheumatol. 2003;30(10):2218-2225.

47. Campbell TM, Trudel G, Laneuville O. Knee flexion contractures in patients with osteoarthritis: clinical features and histologic characterization of the posterior capsule. PMR. 2014;7(5):466-473.
48. Sherer BA, Godlewski KF, Levine LA. Pharmacologic therapy for Peyronie's disease: what should we prescribe? Expert Opin Pharmacother. 2015;16(9):1299-1311.

49. Cheung K, Walley KC, Rozental TD. Management of complications of Dupuytren contracture. Hand Clin. 2015;31(2):345-354.

\section{Publish your work in this journal}

Drug Design, Development and Therapy is an international, peerreviewed open-access journal that spans the spectrum of drug design and development through to clinical applications. Clinical outcomes, patient safety, and programs for the development and effective, safe, and sustained use of medicines are the features of the journal, which has also been accepted for indexing on PubMed Central. The manuscript management system is completely online and includes a very quick and fair peer-review system, which is all easy to use. Visit http://www.dovepress.com/testimonials.php to read real quotes from published authors.

Submit your manuscript here: http://www.dovepress.com/drug-design-development-and-therapy-journal 